\title{
The utility of hyperlactataemia in the definition of septic shock: Evaluating the Sepsis-3 definitions in a sub-Saharan African intensive care unit
}

\author{
A A Elhouni, MB ChB, MSc; K de Vasconcellos, MB ChB, FCA (SA), Cert Crit Care \\ Discipline of Anaesthesiology and Critical Care, School of Clinical Medicine, College of Health Sciences, University of KwaZulu-Natal, \\ Durban, South Africa; and Intensive Care Unit, King Edward VIII Hospital, Durban, South Africa
}

Corresponding author: A A Elhouni(ali.el_houni@yahoo.com)

\begin{abstract}
Background. Sepsis-3 definitions were published in 2016 and included hyperlactataemia (serum lactate $>2.0 \mathrm{mmol} / \mathrm{L}$ ) as a mandatory component of the new definition of septic shock. These data were collected mainly from high-income countries and lack adequate validation in scenarios outside these countries.

Objectives. To evaluate admission serum lactate as a predictor of intensive care unit (ICU) mortality in patients with infection and hypotension requiring inotropic support.

Methods. This was a retrospective observational study of 170 patients with infection and hypotension requiring inotropic support admitted to the ICU at King Edward VIII Hospital in Durban, South Africa. Admission serum lactate was evaluated as a predictor of ICU mortality in this cohort.

Results. The study population had a median age of only 42 years. The ICU mortality rate for the cohort was $49.4 \%$. Most patients were surgical $(71.8 \%)$, with the most common source of sepsis being abdominal (55.9\%). The ICU mortality rate was $40.9 \%$ in patients with a lactate level $\leq 2.0 \mathrm{mmol} / \mathrm{L}$ and $52.4 \%$ in those with a level $>2.0 \mathrm{mmol} / \mathrm{L}$; this did not reach statistical significance. The optimal cut-off was $4.5 \mathrm{mmol} / \mathrm{L}$, at which there was a clear, statistically significant difference in mortality between patients without (39.3\%) and with hyperlactataemia $(59.3 \%)(p=0.009)$.

Conclusions. Hyperlactataemia was associated with increased mortality. However, a lactate level $>2.0 \mathrm{mmol} / \mathrm{l}$, as proposed in Sepsis-3, did not reach statistical significance, and a higher cut-off of $>4.5 \mathrm{mmol} / \mathrm{L}$ was more appropriate.
\end{abstract}

S Afr Med J 2019;109(11):880-884. https://doi.org/10.7196/SAMJ.2019.v109i11.13968

Infectious disease is a major cause of morbidity and mortality, and is responsible for $>50 \%$ of intensive care unit (ICU) admissions globally. ${ }^{[1]}$ Attempts to improve outcomes of patients with sepsis include better definitions of sepsis, identifying high-risk patient subgroups, and allocation of therapies to patient subgroups that are most likely to benefit from these therapies.

Lactate is a product of the metabolism of glucose during glycolysis. It results from the conversion of pyruvate to lactate by the action of lactate dehydrogenase. During sepsis, an increased blood lactate concentration, 'hyperlactataemia, is seen during tissue dysoxia where sufficient oxygen is not available, as in hypoperfusion states, or not utilisable, when mitochondrial oxidation is impaired. ${ }^{[2]}$ It may also result from overstimulation of aerobic glycolysis by inflammatory mediators leading to glycolytic flux that exceeds the capacity of enzymes to convert pyruvate to acetyl coenzyme A. Finally, hyperlactataemia may result from impaired lactate clearance rather than overproduction. ${ }^{[3]}$

In patients with sepsis and septic shock, hyperlactataemia is therefore common, being found in $>50 \%$ of patients, and may be associated with increased morbidity and mortality. ${ }^{[4,5]}$ In patients with severe sepsis and septic shock, hyperlactataemia may be associated with an increased risk of death independent of vasopressor need, especially if the initial lactate level is $>4 \mathrm{mmol} / \mathrm{L} \cdot{ }^{[6,7]}$

The Third International Consensus Definitions for Sepsis and Septic Shock (Sepsis-3) were published in 2016 and included hyperlactataemia (serum lactate $>2.0 \mathrm{mmol} / \mathrm{L}$ ) as a mandatory component of the new definition of septic shock. ${ }^{[8]}$ The rationale for this inclusion was that patients with both hypotension requiring inotropic support and hyperlactataemia represented a subgroup with significantly higher mortality than patients with either abnormality alone. Clinically, the addition of lactate as a mandatory marker in the new definition may alert the clinician to cellular and cardiovascular dysfunction that is not apparent from the need for inotropic support alone..$^{[9]}$

These changes in the definitions of sepsis and septic shock have significant implications for the clinical care of patients with sepsis and for future research in these patients. Despite these definitions being derived from large international databases, much criticism still surrounds them. These data were collected mainly from highincome countries (HICs) and lack adequate validation in scenarios outside these countries. The only study that the authors are aware of outside a HIC was conducted in Brazil. ${ }^{[10]}$ While this study validated the use of hyperlactataemia in identifying a high-risk subset of patients with sepsis and hypotension requiring inotropic support, it suggested that a higher cut-off $(>4.0 \mathrm{mmol} / \mathrm{L})$ was optimal in their setting. It is therefore unknown whether the current Sepsis-3 definition is applicable to all patient populations in low- and middleincome countries (LMICs), ${ }^{[10]}$ and specifically no studies have been performed in sub-Saharan Africa. The critical care data from the South African Surgical Outcomes Study indicated that significant differences exist between South African (SA) critical care patients and those in HICs: they were significantly younger, had a significantly 
lower rate of ICU admission and underwent more urgent surgery than the European Surgical Outcomes Study patients. ${ }^{[11]}$ A recent local publication ${ }^{[12]}$ showed that the spectrum of surgical sepsis presenting to SA hospitals is different from that seen in HICs. Their patient cohort was much younger and generally presented with more advanced disease.

\section{Objectives}

There is both a lack of appropriate research in SA and evidence that regional differences in the applicability of the Sepsis-3 definition may exist. It is therefore essential that the definition of septic shock as proposed in Sepsis-3 is validated in the SA context prior to clinical application. Our objective was to evaluate whether the new Sepsis-3 definition of septic shock, and specifically the requirement for hyperlactataemia in addition to inotropic support, retained utility in the SA context. In particular, we aimed to assess whether hyperlactataemia identified a patient subgroup at significantly higher risk of death than those without hyperlactataemia, and if so, what the optimal cut-off point would be.

\section{Methods}

This study was a retrospective observational chart review of patients admitted to the ICU at King Edward VIII Hospital, an academic tertiary medical centre in Durban, SA. The study ICU is a 12-bed mixed medical/surgical ICU serving the province of KwaZulu-Natal.

The study protocol was approved by the University of KwaZuluNatal Biomedical Research Ethics Committee (ref. no. BE-457/17) and the provincial Department of Health (ref. no. HRKM 475/17).

\section{Data collection}

All data collected were obtained from patient files stored in the ICU. These files contained clinical and laboratory data necessary for the study, which had been collected as part of routine clinical care. Data collection started from the date of ethical approval backward until the calculated sample size was obtained. ICU files of consecutive patients admitted to the ICU were assessed for eligibility into the study. For the purpose of the study, 'inotropic' support was defined as use of either of the 'inopressors' adrenaline or noradrenaline. Pure vasopressors such as vasopressin or phenylephrine are not used in the study ICU, and the 'pure' inotrope dobutamine is not used in the unit in patients in septic shock. All adult patients admitted with hypotension requiring inotropic support, which was believed by the admitting intensivist to be infectious in aetiology ('septic shock' as per consensus definition prior to Sepsis-3), were eligible for inclusion. Children aged $<18$ years and patients who did not have lactate measured were excluded. Admission serum lactate, as measured by the GEM Premier 3000 blood gas machine (Instrumentation Laboratory, Werfen Group, Spain), was used for the purposes of this study.

\section{Statistical analysis}

Sample size was calculated using data from Sepsis-3 that reported a mortality rate of $54.0 \%$ in the hyperlactataemic and inotroperequiring subgroup, and $25.2 \%$ in the subgroup that required inotropic support but was not hyperlactataemic (data from the Pittsburgh Medical Centre). ${ }^{[8]}$ These data were chosen because they best approximated the baseline mortality of patients with septic shock in the study ICU. The alpha value was set at $5 \%$ and the power at $90 \%$, and a 2:1 ratio between patients with and without hyperlactataemia was chosen. The sample size using this methodology was estimated at 129 . Preliminary data suggested a ratio closer to $3: 1$, so the sample size was increased to 170 to allow for uncertainty in this parameter.
Statistical analysis was performed using SPSS Statistics for Windows, version 25.0 (IBM, USA), and R, version 3.5.1. Categorical variables were described as percentages and compared using the $\chi^{2}$ test or Fisher's exact test, where appropriate. Continuous data were described using means and standard deviations when normally distributed and medians and interquartile ranges when the distribution was non-Gaussian. These data were compared using the independentsamples $t$-test or the Mann-Whitney $U$-test, respectively. A receiver operating characteristic (ROC) curve was constructed for lactate. Optimal cut-off points were established for each of the variables by determining the point closest to the $(0.1)$ corner in the ROC plane. Multivariable logistic regression analysis was performed using demographic variables that were significant $(p<0.05)$ on univariate analysis, the admission sequential organ failure assessment (SOFA) score and serum lactate (as a continuous variable). Further analyses were performed where lactate as a continuous variable was replaced with lactate as a categorical variable using the cut-offs of $2.0 \mathrm{mmol} / \mathrm{L}$ (as per Sepsis-3) and the optimal cut-off point identified in the ROC analysis. This was intended as a more clinically applicable analysis, as the use of binary categorical variables is arguably more useful from a clinical point of view, while continuous variables may be preferable statistically. The diagnostic performance (in terms of ICU mortality) of the above cut-offs $(2.0 \mathrm{mmol} / \mathrm{L}$ and the optimal cut-off on ROC analysis) was also evaluated in terms of sensitivity, specificity, positive predictive value, negative predictive value, positive likelihood ratio, negative likelihood ratio, number needed to diagnose and diagnostic accuracy. In addition, the performance of the two key lactate cutoffs was compared using net reclassification improvement (NRI). For this purpose, mortality risk was stratified using the following categories: predicted mortality <25.0\%, $25.0-49.9 \%, 50.0-74.9 \%$ and $\geq 75.0 \%$, which were derived from the multivariable logistic regression analyses described above.

\section{Results}

A total of 170 adult patients with sepsis requiring inotropic support and who had an admission serum lactate result available were included in the study. The ICU mortality rate for the cohort was 49.4\% (84 patients). Most patients received adrenaline, with only 8 (4.7\%) receiving noradrenaline.

Table 1 highlights the demographic and outcome data for the cohort, as well as admission serum lactate data, and provides the univariate analyses for these factors and ICU mortality. Most patients were surgical (71.8\%), with the most common source of sepsis being abdominal (55.9\%), followed by pulmonary (25.9\%).

ROC curve analysis for admission serum lactate and ICU mortality is shown in Fig. 1. The area under the curve was 0.612, standard error 0.041 (95\% confidence interval $0.527-0.696)(p=0.012)$. The optimal cut-off point was $4.5 \mathrm{mmol} / \mathrm{L}$.

Table 2 shows the categorical univariate analyses for various cutoffs for lactate for ICU mortality. The cut-offs for lactate were derived from the Sepsis-3 definition $(>2.0 \mathrm{mmol} / \mathrm{L})$ and other cut-offs explored in the literature $(>3.0 \mathrm{or}>4.0 \mathrm{mmol} / \mathrm{L})$, as well as the optimal cut-off from the ROC curve analysis $(>4.5 \mathrm{mmol} / \mathrm{L})$. Using the cutoff of $2.0 \mathrm{mmol} / \mathrm{L}, 126$ patients $(74.1 \%)$ were hyperlactataemic. ICU mortality was $52.4 \%$ for patients with an admission serum lactate level $>2.0 \mathrm{mmol} / \mathrm{L}$, as opposed to $40.9 \%$ for those with a level $\leq 2.0 \mathrm{mmol} / \mathrm{L}$. The corresponding values for a lactate cut-off of $4.5 \mathrm{mmol} / \mathrm{L}$ were $39.3 \%$ and $59.3 \%$.

The results of the multivariable logistic regression analysis using lactate as continuous variable are shown in Table 3. The additional analyses with lactate as categorical variable (using the cut-offs of 2.0 and $4.5 \mathrm{mmol} / \mathrm{L}$ ) are shown below this in Table 3. Lactate as a 
Table 1. Demographic data, outcomes and admission serum lactate

\begin{tabular}{|c|c|c|c|c|}
\hline & Entire cohort & Survived & Died & $p$-value \\
\hline Age, median (IQR) & $42(30.00-58.00)$ & $38(26.00-53.00)$ & $48(34.00-62.00)$ & $<0.001$ \\
\hline Gender, $n(\%)$ & & & & 0.003 \\
\hline Female & $94(55.3)$ & $38(44.2)$ & $56(66.7)$ & \\
\hline Admission discipline, $n(\%)$ & & & & 0.144 \\
\hline Medicine & $48(28.2)$ & $20(23.3)$ & $28(33.3)$ & \\
\hline Surgery & $122(71.8)$ & $66(76.7)$ & $56(66.7)$ & \\
\hline Source of sepsis, $n(\%)$ & & & & 0.714 \\
\hline Abdominal & 95 (55.9) & $51(59.3)$ & $44(52.4)$ & \\
\hline Bloodstream infection & $3(1.8)$ & $2(2.3)$ & $1(1.2)$ & \\
\hline Chest & $44(25.9)$ & $20(23.3)$ & $24(28.6)$ & \\
\hline CNS & $4(2.4)$ & $1(1.2)$ & $3(3.6)$ & \\
\hline Skin and soft tissue & $24(14.1)$ & $12(14.0)$ & $12(14.3)$ & \\
\hline Duration of ICU stay (days), median (IQR) & $5(3-7)$ & $6(4-8)$ & $3(2-6)$ & $<0.001$ \\
\hline Duration of inotropic support (days), median (IQR) & $3(2-4)$ & $3(2-4)$ & $3(2-4)$ & 0.925 \\
\hline Duration of ventilation (days), median (IQR) & $4(2-6)$ & $4(3-7)$ & $3(2-6)$ & 0.072 \\
\hline SOFA (admission), median (IQR) & $10(8-12)$ & $9(7-11)$ & $11(9-12)$ & 0.003 \\
\hline Renal replacement therapy in ICU, $n(\%)$ & $24(14.1)$ & $13(15.1)$ & $11(13.1)$ & 0.705 \\
\hline Ventilation in ICU, $n(\%)$ & $167(98.2)$ & $83(96.5)$ & $84(100)$ & 0.246 \\
\hline Admission serum lactate (mmol/L), median (IQR) & $4.6(2-7.5)$ & $3.8(1.9-6.8)$ & $5.6(2.6-8.2)$ & 0.012 \\
\hline
\end{tabular}

Table 2. Univariate analyses of lactate categorical variables for intensive care unit mortality

\begin{tabular}{|c|c|c|c|c|c|}
\hline Lactate level $(\mathrm{mmol} / \mathrm{L})$ & Entire cohort, $n(\%)$ & Survived, $n(\%)$ & Died, $n(\%)$ & $p$-value & OR (95\% CI) \\
\hline$>2.0$ & $126(74.1)$ & $60(69.8)$ & $66(78.6)$ & 0.190 & $1.59(0.79-3.19)$ \\
\hline$>3.0$ & $112(65.9)$ & $50(58.1)$ & $62(73.8)$ & 0.031 & $2.03(1.06-3.88)$ \\
\hline$>4.0$ & $93(54.7)$ & $40(46.5)$ & $53(63.1)$ & 0.030 & $1.97(1.07-3.63)$ \\
\hline$>4.5$ & $86(50.6)$ & $35(40.7)$ & $51(60.7)$ & 0.009 & $2.25(1.22-4.16)$ \\
\hline
\end{tabular}

$\mathrm{OR}=$ odds ratio $\mathrm{CI}=$ confidence interval.

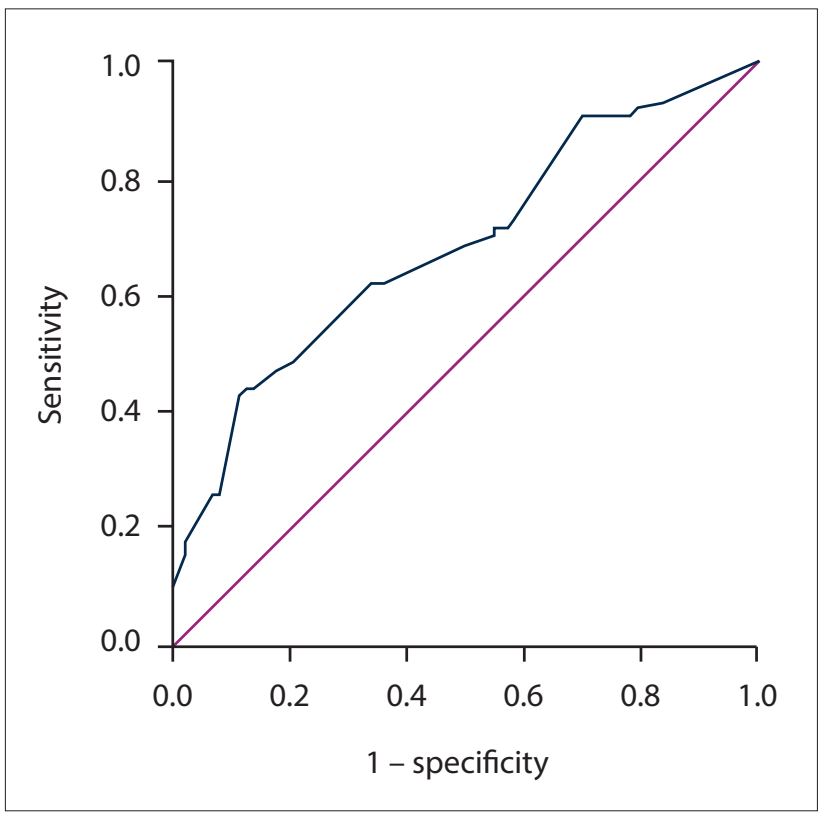

Fig. 1. Receiver operating characteristic curve of serum lactate and intensive care unit mortality.

continuous variable and as a categorical variable with a cut-off of $4.5 \mathrm{mmol} / \mathrm{L}$ remained independently associated with ICU mortality.

\begin{tabular}{|c|c|c|c|}
\hline & & OR $(95 \% \mathrm{CI})$ & $p$-value \\
\hline \multirow[t]{4}{*}{1} & Age & $1.04(1.02-1.06)$ & 0.001 \\
\hline & Gender (female) & $2.35(1.19-4.67)$ & 0.014 \\
\hline & Lactate & $1.13(1.02-1.25)$ & 0.019 \\
\hline & SOFA & $1.15(1.02-1.30)$ & 0.028 \\
\hline \multirow[t]{4}{*}{2} & Age & $1.03(1.01-1.06)$ & 0.001 \\
\hline & Gender (female) & $2.41(1.22-4.73)$ & 0.011 \\
\hline & Lactate $>2.0 \mathrm{mmol} / \mathrm{L}$ & $1.35(0.63-2.86)$ & 0.439 \\
\hline & SOFA & $1.16(1.03-1.31)$ & 0.015 \\
\hline \multirow[t]{4}{*}{3} & Age & $1.04(1.02-1.06)$ & 0.001 \\
\hline & Gender (female) & $2.41(1.22-4.78)$ & 0.011 \\
\hline & Lactate $>4.5 \mathrm{mmol} / \mathrm{L}$ & $2.26(1.14-4.52)$ & 0.020 \\
\hline & SOFA & $1.15(1.01-1.29)$ & 0.030 \\
\hline
\end{tabular}

$\mathrm{OR}=$ odds ratio; $\mathrm{CI}=$ confidence interval; $\mathrm{SOFA}=$ sequential organ failure assessment.

The diagnostic performance of lactate as a categorical variable with the key cut-offs of $2.0 \mathrm{mmol} / \mathrm{L}$ and $4.5 \mathrm{mmol} / \mathrm{L}$ is shown in Table 4.

The net reclassification improvement for a lactate cut-off of $4.5 \mathrm{mmol} / \mathrm{L}$ v. $2.0 \mathrm{mmol} / \mathrm{L}$ was $5.0 \%$ for those with events (ICU mortality), while the NRI for those without events (ICU survival) was $8.2 \%$, giving a total NRI of $13.2 \%(p=0.104)$. 
Table 4. Diagnostic performance of lactate as a categorical variable

\begin{tabular}{|c|c|c|c|c|c|c|c|c|}
\hline $\begin{array}{l}\begin{array}{l}\text { Lactate } \\
(\mathrm{mmol} / \mathrm{L})\end{array} \\
\end{array}$ & $\begin{array}{l}\text { Sensitivity, } \\
\%(95 \% \mathrm{CI})\end{array}$ & $\begin{array}{l}\text { Specificity, } \\
\%(95 \% \mathrm{CI})\end{array}$ & $\begin{array}{l}\text { PPV, \% } \\
(95 \% \text { CI })\end{array}$ & $\begin{array}{l}\text { NPV, \% } \\
(95 \% \mathrm{CI})\end{array}$ & $\begin{array}{l}\text { PLR } \\
(95 \% \text { CI })\end{array}$ & $\begin{array}{l}\text { NLR } \\
(95 \% \text { CI })\end{array}$ & NND & $\begin{array}{l}\text { Diagnostic accuracy, } \\
\%(95 \% \mathrm{CI})\end{array}$ \\
\hline$>2.0$ & $\begin{array}{l}78.6(68.3- \\
86.8)\end{array}$ & $\begin{array}{l}30.2(20.8- \\
41.1)\end{array}$ & $\begin{array}{l}52.4(43.3- \\
61.4)\end{array}$ & $\begin{array}{l}59.1(43.3- \\
73.7)\end{array}$ & $\begin{array}{l}1.13(0.94- \\
1.35)\end{array}$ & $\begin{array}{l}0.71(0.42- \\
1.19)\end{array}$ & 11.4 & $54.2(46.3-61.8)$ \\
\hline$>4.5$ & $\begin{array}{l}60.7(49.5- \\
71.2)\end{array}$ & $\begin{array}{l}59.3(48.2- \\
69.8)\end{array}$ & $\begin{array}{l}59.3(48.2- \\
69.8)\end{array}$ & $\begin{array}{l}60.7(49.5- \\
71.2)\end{array}$ & $\begin{array}{l}1.49(1.10- \\
2.03)\end{array}$ & $\begin{array}{l}0.66(0.48- \\
0.91)\end{array}$ & 5 & $60.6(52.2-67.4)$ \\
\hline
\end{tabular}

$\mathrm{PPV}=$ positive predictive value; $\mathrm{NPV}=$ negative predictive value; $\mathrm{PLR}=$ positive likelihood ratio; $\mathrm{NLR}=$ negative likelihood ratio; $\mathrm{NND}=$ number needed to diagnose; $\mathrm{CI}=$ confidence interval.

\section{Discussion}

For decades, hyperlactataemia has been shown to be associated with increased mortality in patients with shock, and in particular septic shock. ${ }^{[7,13]}$ Lactate has been used as a marker of 'cryptic' shock, and lactate clearance has been used to guide resuscitation. ${ }^{[14-16]}$ Recently the use of lactate to risk-stratify patients with sepsis, and sepsis-induced hypotension requiring inotropic support, has been advocated in the Sepsis-3 consensus definitions of sepsis and septic shock. EPIC II showed significant international differences in the prevalence of infections, types of infecting micro-organisms, and mortality rates in Eastern Europe compared with Western Europe, in Australia compared with Asia, and in Latin America compared with North America. ${ }^{[17,18]}$ The present study is the first to evaluate the use of lactate to risk-stratify patients with sepsis-induced hypotension requiring inotropic support in sub-Saharan Africa.

The study population was young, with a median age of only 42 years. This contrasts with most international ICU literature and illustrates the importance of validating international guidelines in the SA context. ${ }^{[11]}$ While there was a preponderance of surgical patients and patients with intra-abdominal sepsis in the study, the cohort comprised a spectrum of patients, a significant number of whom had pneumonia and skin and soft-tissue infections. ${ }^{[12]}$ As expected of a cohort of patients in septic shock, mortality was relatively high at $49.4 \%$. This figure is in keeping with the median admission SOFA score of 10 . The median ICU length of stay was relatively short at 5 days, reflecting pressure on ICU beds in the study ICU.

The rationale for the addition of serum lactate to the definition of septic shock in Sepsis-3 was to allow for the differentiation of patients with sepsis-induced hypotension into high-risk and low-risk subgroups. As such, patients in the cohorts reported in Sepsis-3 who had hypotension requiring inotropic support and elevated serum lactate had higher mortality than those without elevated serum lactate. Patients without hyperlactataemia had hospital mortalities of $18.8 \%, 25.2 \%$, and $30.1 \%$ in the three cohorts evaluated, and mortality rates increased to $35 \%, 42.3 \%$, and $54 \%$, respectively, in patients with a serum lactate level of $>2.0 \mathrm{mmol} / \mathrm{L} .^{[8]}$

The objective of this study was to determine whether hyperlactataemia in patients with sepsis requiring inotropic support was also associated with increased mortality in the SA context. Neither the derivation nor validations cohorts for the Sepsis-3 definitions appear to have included any patients from the African continent. As with the results reported in Sepsis-3, hyperlactataemia was associated with increased mortality in our study. While the mortality rate in our study was $40.9 \%$ in patients with a lactate level of $\leq 2.0 \mathrm{mmol} / \mathrm{L}$ and $52.4 \%$ in those with a lactate level of $>2.0 \mathrm{mmol} / \mathrm{L}$, this difference did not reach statistical significance. In our study the optimal cut-off was $4.5 \mathrm{mmol} / \mathrm{L}$, which is similar to the optimal cutoff of $4.0 \mathrm{mmol} / \mathrm{L}$ in a Brazilian study. At a cut-off of $4.5 \mathrm{mmol} / \mathrm{L}$ there was a clear, statistically significant difference in mortality between patients without hyperlactataemia (39.3\%) and those with hyperlactataemia $(59.3 \%)(p=0.009)$.
It is noteworthy that the SA and Brazilian cohorts (median age 42 years and 52 years, respectively) were younger than the Sepsis-3 cohorts ( $>60$ years).$^{[8,10]} \mathrm{A}$ recent publication compared the prognostic value of hyperlactataemia in predicting mortality between elderly ( $\geq 65$ years) and non-elderly ( $<65$ years) patients with sepsis, and showed that elderly non-survivors had a $1 \mathrm{mmol} / \mathrm{L}$ lower serum lactate level than non-elderly non-survivors. ${ }^{[19]}$ One explanation is that elderly patients have different physiology and multiple systemic disorders compared with younger adults. Elderly patients with sepsis had a lower temperature, lower heart rate and higher mean arterial pressure, which may alter their lactate production and metabolism. ${ }^{[19]}$ Other explanations as to why patients with sepsis from HICs had lower lactate levels than patients from LMICs include the use of early protocolised sepsis management and resuscitation in HICs that relies on complex invasive technology that may not be widely available in resource-limited countries. ${ }^{[20]}$ This early sepsis recognition and resuscitation can augment lactate clearance, which may be reflected as lower lactate levels in these patients on ICU admission. The need for a higher lactate threshold in our study could also have been due to the predominant use of adrenaline in the study ICU, which may theoretically lead to an increase in lactate levels. ${ }^{[21]}$ While our study had a predominance of patients with abdominal sepsis, both the Brazilian and Sepsis-3 cohorts had a predominance of patients with pneumonia. This difference has been observed in previous studies, where elderly patients with sepsis were more likely to have respiratory tract infection, while non-elderly patients were more likely to have abdominal and soft-tissue sepsis. ${ }^{[1,22]}$

While the present study broadly validates the principle that hyperlactataemia identifies a particularly high-risk subset of patients with sepsis who require inotropic/vasopressor support, the concern remains that the use of lactate measurement in this setting may impose unnecessary financial or logistical costs, or that it may simply not be available in certain settings. While the study ICU is in a middle-income country, it is comparatively well-resourced and the availability of lactate measurements cannot be taken for granted in other middle- and lower-income settings. For this reason, further research is required to evaluate the utility of clinical parameters that may provide equivalent risk-stratification benefits.

\section{Study limitations}

This study has several potential limitations. The retrospective design could have led to bias. The data were derived from a single ICU, and may not be generalisable. The study ICU is a busy referral centre, however, and is likely to be representative of the realities of critical care in at least the state sector in SA. It is the only study of its kind from Africa and is therefore the most representative study available for the continent. The primary outcome was ICU mortality as opposed to hospital mortality, which is the most common outcome used in similar studies. The choice of ICU mortality was due to practical difficulties in establishing hospital mortality in the study population, and while this does not invalidate the findings of this 
study, it does potentially confound direct comparison with other studies that used hospital mortality as their primary endpoint. The use of adrenaline as the predominant inotrope/vasopressor may have led to an increase in serum lactate levels. Noradrenaline is not freely available in SA, further illustrating the need for studies that reflect practice in different settings.

\section{Conclusions}

In our study, hyperlactataemia was associated with increased mortality. However, a lactate level $>2.0 \mathrm{mmol} / \mathrm{L}$, as proposed in Sepsis-3, did not reach statistical significance. A higher cut-off of $>4.5 \mathrm{mmol} / \mathrm{L}$ should be used in the SA context (and potentially in other LMICs) to identify patients with sepsis-induced hypotension requiring inotropic support who are at increased risk of death.

Declaration. Publication of this article was a requirement for AAE's MMed (Anaesthetics) degree.

Acknowledgements. None.

Author contributions. AAE: study design, data collection, drafting and revision of manuscript. KdV: study design data analysis, drafting and revision of manuscript.

Funding. None.

Conflicts of interest. None.

1. Vincent JL, Marshall JC, Namendys-Silva SA, et al. Assessment of the worldwide burden of critical illness: The intensive care over nations (ICON) audit. Lancet Respir Med 2014;2(5):380-386. https://doi. $\mathrm{rg} / 10.1016 /$ S2213-2600(14)70061-X

2. Suetrong B, Walley KR. Lactic acidosis in sepsis: It's not all anaerobic: Implications for diagnosis and management. Chest 2016;149(1):252-261. https://doi.org/10.1378/chest.15-1703

3. Kraut JA, Madias NE. Lactic acidosis. N Engl J Med 2014;371(24):2309-2319. https://doi.org/10.1056/ NEJMc1500327

4. Dugas AF, Mackenhauer J, Salciccioli JD, Cocchi MN, Gautam S, Donnino MW. Prevalence and characteristics of nonlactate and lactate expressors in septic shock. J Crit Care 2012;27(4):344-350. https://doi.org/10.1016/j.jcrc.2012.01.005
5. Casserly B, Phillips GS, Schorr C, et al. Lactate measurements in sepsis-induced tissue hypoperfusion: Results from the Surviving Sepsis Campaign Database. Crit Care Med 2015;43(3):567-573. https://doi. Results from the Surviving Sepsis Can

6. Thomas-Rueddel DO, Poidinger B, Weiss M, et al. Hyperlactatemia is an independent predictor of Thomas-Rueddel DO, Poidinger B, Weiss M, et al. Hyperlactatemia is an independent predictor of
mortality and denotes distinct subtypes of severe sepsis and septic shock. J Crit Care 2015;30(2):439. mortality and denotes distinct subtypes of severe sepsis

el-439.e6. https://doi.org/10.1016/j.jcrc.2014.10.027
7. Trzeclak S, Dellinger RP, Chansky ME, et al. Serum lactate as a predictor of mortality in patients with Trzeclak S, Dellinger RP, Chansky ME, et al. Serum lactate as a predictor of mortality in pa-
infection. Intensive Care Med 2007;33(6):970-977. https://doi.org/10.1007/s00134-007-0563-9

8. Singer M, Deutschman CS, Seymour CW, et al. The Third International Consensus Definitions for Sepsis and Septic Shock (Sepsis-3). JAMA 2016;315(8):801-810. https://doi.org/10.1001/jama.2016.028

9. Cecconi M, de Backer D, Antonelli M, et al. Consensus on circulatory shock and hemodynamic monitoring. Task Force of the European Society of Intensive Care Medicine. Intensive Care Med 2014:40(12):1795-1815. https://doi.org/10.1007/s00134-014-3525-z

10. Besen BA, Romano TG, Nassar AP, et al. Sepsis-3 definitions predict ICU mortality in a low-middleincome country. Ann Intensive Care 2016;6(1):107. https://doi.org/10.1186/s13613-016-0204-y

11. Biccard BM, Madiba TE. The South African Surgical Outcomes Study: A 7-day prospective observational cohort study. S Afr Med J 2015;105(6):465-475. https://doi.org/10.7196/SAMJ.9435

12. Green S, Kong VY, Clarke DL, et al. The spectrum and outcome of surgical sepsis in Pietermaritzbur South Africa. S Afr Med J 2017;107(2):134-136. https://doi.org/10.7196/SAMJ.2017.v107i2.11339

13. Broder G, Weil MH. Excess lactate: An index of reversibility of shock in human patients. Science 1964;143(3613):1457-1459. https://doi.org/10.1126/science.143.3613.1457

14. Jansen TC, van Bommel J, Mulder PG, et al. The prognostic value of blood lactate levels relative to that of vital signs in the pre-hospital setting: A pilot study. Crit Care 2008;12:R160. https://doi.org/10.1186/ cc7159

15. Eastridge BJ, Salinas J, McManus JG, et al. Hypotension begins at $110 \mathrm{~mm}$ Hg: Redefining 'hypotension' with data. J Trauma 2007;63(2):291-297. https://doi.org/10.1097/TA.0b013e31809ed924

16. Jansen TC, van Bommel J, Schoonderbeek FJ, et al; LACTATE Study Group. Early lactate-guided therapy in intensive care unit patients: A multicenter, open-label, randomized controlled trial. Am J Respir Crit Care Med 2010;182(6):752-761. https://doi.org/10.1164/rccm.200912-19180C

17. Vincent JL, Rello J, Marshall J, et al. International study of the prevalence and outcomes in infection in intensive care units. JAMA 2009;302(21):2323-2329. https://doi.org/10.1001/jama.2009.1754

18. Sartelli S, Kluger Y, Ansaloni L, et al. Raising concerns about the Sepsis-3 definitions. World J Emerg Surg 2018;13:6. https://doi.org/10.1186/s13017-018-0165-6

19. Cheng HH, Chen FC, Change MW, et al. Difference between elderly and non-elderly patients in using serum lactate level to predict mortality caused by sepsis in the emergency department. Medicine 2018:97(13):e0209. https://doi.org/10.1097/MD.0000000000010209

20. Becker JU, Theodosis C, Jacob ST, et al. Surviving sepsis in low-income and middle-income countries: New directions for care and research. Lancet Infect Dis 2009;9(9):577-582. https://doi.org/10.1016 S1473-3099(09)70135-5

21. Levy B. Bench-to-bedside review: Is there a place for epinephrine in septic shock? Critical Care 2005;9:561-565. https://doi.org/10.1186/cc3901

22. Martin GS, Mannino DM, Moss M. The effect of age on the development and outcome of adult sepsis. Crit Care Med 2006;34(1):15-21. https://doi.org/10.1097/01.CCM.0000194535.82812.BA 